\title{
Evolution of the nitric oxide synthase family in vertebrates and novel insights in gill development
}

(1)

(6)

(1)

(1)

${ }^{1}$ Biology and Evolution of Marine Organisms, Stazione Zoologica Anton Dohrn, 80121,

Napoli, Italy

${ }^{2}$ Laboratory for Evolutionary Morphology, RIKEN Center for Biosystems Dynamics

Research (BDR), Kobe, 650-0047, Japan

${ }^{3}$ Evolutionary Morphology Laboratory, RIKEN Cluster for Pioneering Research (CPR), 2-2-

3 Minatojima-minami, Chuo-ku, Kobe, Hyogo, 650-0047, Japan

${ }^{4}$ Department of Integrative Biology and Program in Ecology, Evolution \& Behavior (EEB),

Michigan State University, East Lansing, MI 48824, USA

${ }^{5}$ Department of Neuroscience, Spinal Cord and Brain Injury Research Center, and

Ambystoma Genetic Stock Center, University of Kentucky, Lexington, Kentucky, USA

${ }^{6}$ Department of Zoology, Faculty of Science, Charles University in Prague, Prague, Czech

Republic

${ }^{7}$ Division of Biology and Biological Engineering, California Institute of Technology,

Pasadena, CA, USA

${ }^{8}$ South Bohemian Research Center of Aquaculture and Biodiversity of Hydrocenoses,

3 Faculty of Fisheries and Protection of Waters, University of South Bohemia in Ceske

4 Budejovice, Vodnany, Czech Republic

${ }^{9}$ Institute of Neuroscience, University of Oregon, Eugene, OR 97403, USA

6 'Present address: Department of Animal Biology, Faculty of Sciences, University of

7 Málaga; and Andalusian Centre for Nanomedicine and Biotechnology (BIONAND),

8 Málaga, Spain 29

* Correspondence: salvatore.daniello@szn.it 


\section{ORCID}

34 GA: 0000-0001-7806-6761

35 JPA: 0000-0003-3429-9453

36 IB: 0000-0003-4766-611X

37 JS: $0000-0002-3740-3378$

38 VS: 0000-0002-1914-283X

39 SK: 0000-0001-9717-7221

40 JHP: 0000-0002-5476-2137

41 SDA: 0000-0001-7294-1465

42

43

44

45

46

47

48

49

50

51

52

53

54

55

56

57 


\section{(} 8 9 0

\section{Abstract}

Nitric oxide (NO) is an ancestral key signaling molecule essential for life and has enormous versatility in biological systems, including cardiovascular homeostasis, neurotransmission, and immunity. Although our knowledge of nitric oxide synthases (Nos), the enzymes that synthesize NO in vivo, is substantial, the origin of a large and diversified repertoire of nos gene orthologs in fish with respect to tetrapods remains a puzzle. The recent identification of nos3 in the ray-finned fish spotted gar, which was considered lost in the ray-finned fish lineage, changed this perspective. This prompted us to explore nos gene evolution and expression in depth, surveying vertebrate species representing key evolutionary nodes. This study provides noteworthy findings: first, nos2 experienced several lineage-specific gene duplications and losses. Second, nos3 was found to be lost independently in two different teleost lineages, Elopomorpha and Clupeocephala. Third, the expression of at least one nos paralog in the gills of developing shark, bichir, sturgeon, and gar but not in arctic lamprey, suggest that nos expression in this organ likely arose in the last common ancestor of gnathostomes. These results provide a framework for continuing research on nos genes' roles, highlighting subfunctionalization and reciprocal loss of function that occurred in different lineages during vertebrate genome duplications. (6)

\section{(1)}

1 Keywords: Vertebrate evolution; Genome duplication; Gene duplication and loss; NOS;

Phylogenomics; Synteny. 


\section{Introduction}

Originally classified as a pollutant, nitric oxide (NO) was recognized as "Molecule of the Year" in 1992 [1] when its important role as a cellular signaling molecule was recognized. NO plays a role in a myriad of physiological processes, such as cardiovascular homeostasis [2], neurotransmission [3], immune response [4], and in pathological conditions such as neurodegenerative diseases [5] and cancer [6].

Nitric oxide synthase (Nos), the enzyme catalysing the biosynthesis of NO in vivo, is ubiquitous among organisms, including protists and bacteria $[7,8]$. Three nos gene paralogs have been described in vertebrates: two constitutively expressed genes, including nos1 (also known as neuronal nos, or $n$ Nos), which represents the predominant source of $\mathrm{NO}$ involved in neurogenesis and neurotransmission $[9,10]$, and nos3 (endothelial nos or eNos) implicated in angiogenesis and blood pressure control in vascular endothelial cells [11,12]. In addition, nos2 (inducible nos or iNos), which expression is instead evoked by proinflammatory cytokines, is promptly activated in a range of acute stress responses [13].

Although the availability of current genomic data covers all major ray-finned fish lineages, the evolutionary history of their nos gene repertoire remains puzzling. Previous studies reported a variable number of nos genes in teleost fishes: nos1 is always present in a single copy; nos2 either in one or two copies, probably due to the additional teleostspecific whole-genome duplication (TGD) [14-17], or absent as observed in several species. On the other hand, nos3 has been reported as missing in the genomes of rayfinned fish. This apparent gene loss contrasts with literature describing a putative Nos3like protein localized by antibody stains in gills and vascular endothelium of several teleost species $[18,19]$. The discovery of a nos3 ortholog in the spotted gar Lepisosteus oculatus, a holostean fish (the sister group of teleosts within the ray-finned lineage) [20], and the 
108 variable number of teleost nos2 genes raises new questions about the evolution of this

109 important gene family, including: $i$. is our current view on the origin and evolution of nos

110 gene family in vertebrates accurate?; and ii. can further investigation of nos expression

111 pattern in fish retaining a nos3 copy reveal novel functional insights? In an attempt to 112 answer these questions, we have studied the Nos family repertoire at unprecented 113 phylogenetic resolution, investigated conserved syntenies in fish genomes, and studied 114 the expression pattern of all three nos genes during development in multiple species 115 representing key nodes in vertebrate evolution.

\section{Results}

118 Revised evolutionary history of Nos2 and Nos3

119 Gaps in our current knowledge of Nos family evolution include the time of origin of the 120 three distinct paralogous nos genes and when some of them were secondarily lost in 121 specific lineages. Using sequences retrieved from public genomic and transcriptomic 122 databases, we reconstructed a Nos phylogeny using 108 protein sequences from 53 123 species (see Supplementary Table 1). Species were chosen to provide a broad 124 representation of aquatic vertebrates: cyclostomes (modern jawless fish), chondrichthyans 125 (cartilaginous fish), and osteichthyes (bony fish) including ray- and lobe-finned fishes. 126 Lobe-finned fishes include coelacanths, lungfishes, and tetrapods; Ray-finned fishes 127 comprise the non-teleost lineages of polypteriformes (e.g. bichir), acipenseriformes (e.g. 128 sterlet sturgeon), holosteans (lepisosteiformes, e.g. spotted gar, and amiiformes, e.g. 129 bowfin), and the teleosts, subdivided into three major living lineages: osteoglossomorphs 130 (e.g. arowana, mooneyes, and the freshwater elephantfish), elopomorphs (e.g. eels and 131 relatives) and clupeocephalans (e.g. zebrafish and medaka) (see [21] for a recent 132 phylogeny of ray-finned fishes). 
133 Our phylogenetic analysis confirmed that Nos1 is present in all species of jawed

134 vertebrates examined (Fig. 1a, green shading). In contrast, most fish lineages retained 135 Nos2, including chondrichthyans (Callorhinchus milii, Rhincodon typus, Chiloscyllium 136 punctatum, and Scyliorhinus torazame), polypteriformes (Polypterus senegalus, 137 Erpetoichthys calabaricus), acipenseriformes (Acipenser ruthenus), holosteans (Amia 138 calva, Lepisosteus oculatus), elopomorphs (Megalops cyprinoides), osteoglossomorphs 139 (Paramormyrops kingsleyae, Scleropages formosus) and coelacanthiformes (Latimeria 140 chalumnae) (Fig. 1a, grey shading), although a nos2 gene loss event occurred at the stem 141 of Neoteleostei (Fig. 1b), since this gene has not been found in any available genome from 142 this clade. On the other hand, our phylogenetic analysis highlights the occurrence of extra 143 nos2 duplicates in several lineages, for which we adopted a specific nomenclature: in the 144 zebrafish Danio rerio there are two nos2 genes, nos $2 a$ and nos $2 b$, while in the goldfish 145 Carassius auratus, the blind golden-line barbel Sinocyclocheilus anshuiensis and the 146 common carp Cyprinus carpio we found three: nos $2 a$, nos $2 b a$, and nos $2 b b$; in salmonids 147 (Salmo salar and Oncorhynchus mykiss) there are two different copies of nos2, named 148 nos $2 \alpha$ and nos $2 \beta$; and last, we named nos2.1 and nos 2.2 the two nos 2 paralogs that we 149 found in a characid (the Mexican tetra Astyanax mexicanus), a gymnotid (the electric eel 150 Electrophorus electricus), an ictalurid (the channel catfish Ictalurus punctatus), an esocid 151 (the northern pike Esox lucius), and a clupeid (the Atlantic herring Clupea harengus) (Fig. 152 1a, grey shading). Our nomenclature is based both on the phylogenetic analysis and a 153 synteny conservation analysis (see below and in the Discussion section).

154 Nos3 deserves special attention since it was previously believed that a loss event 155 predated the lineage of actinopterygians or alternatively that it represents an innovation of 156 tetrapods [8]. Nevertheless, this latter hypothesis may have been overinterpreted since 157 few ray-finned genome sequences were originally available. The only actinopterygian nos3 
158 gene reported thus far is in the spotted gar [20]. Here we report the identification of nos3 159 genes in genomes of the bichir $P$. senegalus, the sterlet sturgeon $A$. ruthenus [22], the 160 bowfin $A$. calva [23], and the freshwater elephantfish $P$. kingsleyae [24] (Fig. 1a, red 161 shading). The absence of nos3 in all available clupeocephalans indicates a gene loss 162 event in the stem of this group (Fig. 1c). Furthermore, we did not find nos3 in the tarpon $M$. 163 cyprinoides, the most complete genome available among Elopomorpha, nor in 164 transcriptomic data of the European eel Anguilla anguilla. On the other hand, we did 165 identify a nos3 ortholog in the cloudy catshark S. torazame, suggesting its presence in the 166 ancestor of gnathostomes. Previously, two nos genes had been found in the lamprey, 167 called nos $A$ and nosB [8], with unresolved orthology to gnathostome nos1-nos2-nos3, and 168 derived from a lineage-specific tandem duplication in the lamprey lineage. Based on this 169 finding, we searched for the presence of nos genes in other cyclostomes. In the genome of 170 the arctic lamprey Lethenteron camtschaticum [25] we found orthologous genes to $P$. 171 marinus nosA and nosB paralogs. On the other hand, in the inshore hagfish Eptatretus 172 burgeri we identified a single nos gene. Our phylogenetic analysis shows that the hagfish 173 Nos remains outside lamprey NosA-NosB clade, therefore with no clear orthology 174 relationship to any specific gnathostome Nos1, Nos2, Nos3, and suggesting that the 175 duplication giving rise to the lamprey nos $A$-nosB occurred at least before the last common 176 ancestor of Petromyzontidae. 


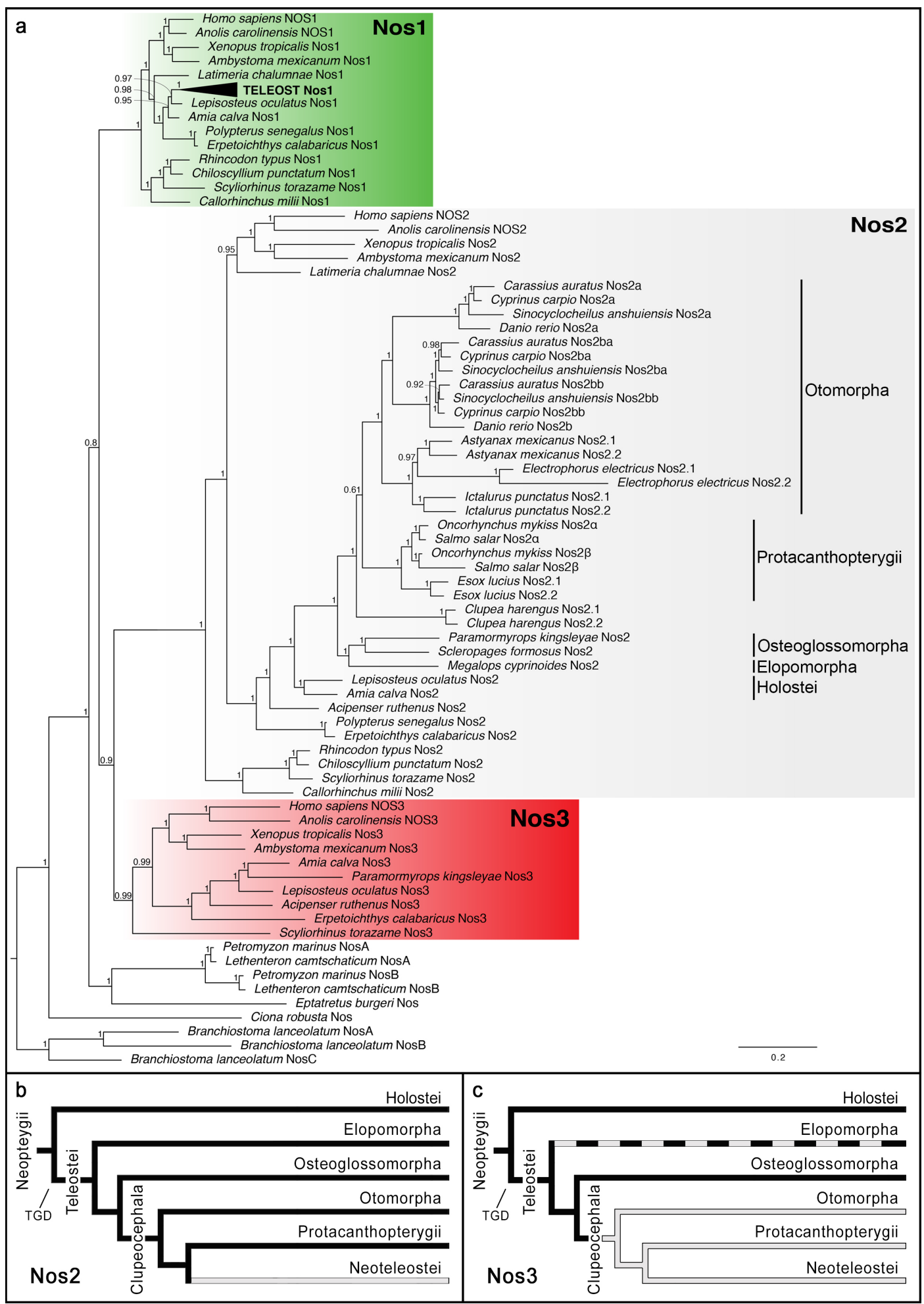

178 Figure 1. Evolution of the Nos gene family. a, Bayesian inference phylogenetic analysis 179 of Nos proteins in chordates. Nos1 clade is indicated by a green shading; light grey 180 shading for the Nos2 clade, and red shading for the Nos3 clade. Numbers at nodes 181 represent posterior probability values. Nos proteins from invertebrate chordates, the 182 lancelet Branchiostoma lanceolatum, and the tunicate Ciona robusta, were used as 
183 outgroup sequences. b-c, Evolutionary scenarios indicating the loss of Nos2 event in 184 Neoteleostei (b) and Nos3 in Clupeocephala (c) as grey lines. Nos3 in Elopomorpha is 185 absent although parsimony suggests it was present in stem elopomorphs, and it is 186 indicated with a dashed line. The schematic representation of Nos1 was omitted because 187 it is present in single copy in all analysed gnathostome species. TGD stands for Teleost188 specific Genome Duplication.

191 In order to better understand the gene loss and expansion events depicted by our 192 phylogenetic analysis, we next analysed the microsynteny (genes linked in proximity) of 193 nos genes in different species. This revealed a complex evolutionary scenario for nos2 194 compared to nos1 and nos3. Specific nos2 duplications in different lineages are explained 195 by distinct evolutionary events in teleosts (Fig. 2a and Supplementary Figure 1). First, the lack of synteny conservation between nos $2 a$ and nos $2 b$ in cyprinids, and the lack of nos $2 a$ in the expected location in non-cyprinid fishes (Supplementary Figure 1) indicates that these paralogs originated in a specific gene duplication event in a common ancestor of the 199 lineage, independently from the TGD (the alternative explanation would require numerous nos $2 a$ losses in several fish lineages), in which while nos $2 b$ has remained the ancestral genomic location, nos2a has been translocated to a different position in the genome (Fig. 2a and Supplementary Fig. 1). Second, an additional genome duplication event after the TGD specifically occurred independently in several teleost lineages, causing the presence 204 of extra nos2 paralogs. These include some cyprinids, in which a carp-specific genome 205 duplication event (Cs4R) likely occurred before the divergence of C. auratus, S. anshuiensis and C. carpio [26], and salmonids (salmonid-specific genome duplication or 207 Ss4R) [27,28], with S. salar and O. mykiss in this study. These additional tetraploidization 208 events can explain the origin of the two independent sets of nos 2 genes in cyprinid and 209 salmonid species. In the case of cyprinids, both our phylogenetic and synteny analyses 
clearly show their nos $2 b$ orthology, and we denote them as nos $2 b a$ and nos $2 b b$ (Fig. $1 a$

211 and Fig. 2a). In the case of salmonids, we name them nos $2 \alpha$ and nos $2 \beta$ to distinguish

212 them from the cyprinid nos2a and nos $2 b$ paralogs, which have a separate origin (see 213 above; Fig. 2a). Third, independent tandem gene duplications explain the presence of two 214 nos2 copies, that we named nos2.1 and nos2.2, located next to each other in the same 215 chromosomal fragment in the genomes of the Atlantic herring (C. harengus), the Mexican 216 tetra (cavefish, A. mexicanus), the electric eel (E. electricus), the channel catfish ( $I$. 217 punctatus), and the northern pike (E. lucius) (Fig. 2a).

218 Bichir, reedfish, sterlet, spotted gar, bowfin and freshwater elephantfish are the only ray219 finned fishes that retained a nos3 ortholog. Therefore, we investigated the absence of 220 nos3 in clupeocephalans. First, we looked for the genomic region containing nos 3 in fishes 221 that represent outgroups to the clupeocephalans. We found one long scaffold of the $P$. kingsleyae genome (scaffold 217) [24] showing extensive conserved synteny with the nos3-containing segment of the linkage group 11 (LG) in the spotted gar genome (Fig. 2b). While these appear to correspond to one of the TGD ohnologons (Fig. 2b), there are other two $P$. kingsleyae scaffold segments (from scaffolds 72 and 104) that together seem to represent the second TGD ohnologon, but lacking the expected nos3 TGD ohnolog (Fig. 2b). Zebrafish chromosomes 16 and 19 and medaka chromosomes 11 and 16 contain orthologous regions to the two $P$. kingsleyae and $L$. oculatus TGD ohnologons, but lack a nos3 gene at the expected locations. One-to-one relationship between these $P$. kingsleyae 230 scaffolds and zebrafish and medaka chromosomes is challenging to determine (Fig. 2b). 231 Regardless, the most parsimonious explanation for the nos3 repertoire in ray-finned fishes 232 is that, first, one of the two nos3 TGD ohnologs was lost in the teleost common ancestor, 233 while the other was retained and later lost in secondary, independent events in the 
234 common ancestor of Clupeocephala and, probably, that of Elopomorpha (Fig. 1C and Fig.

$2352 b)$.

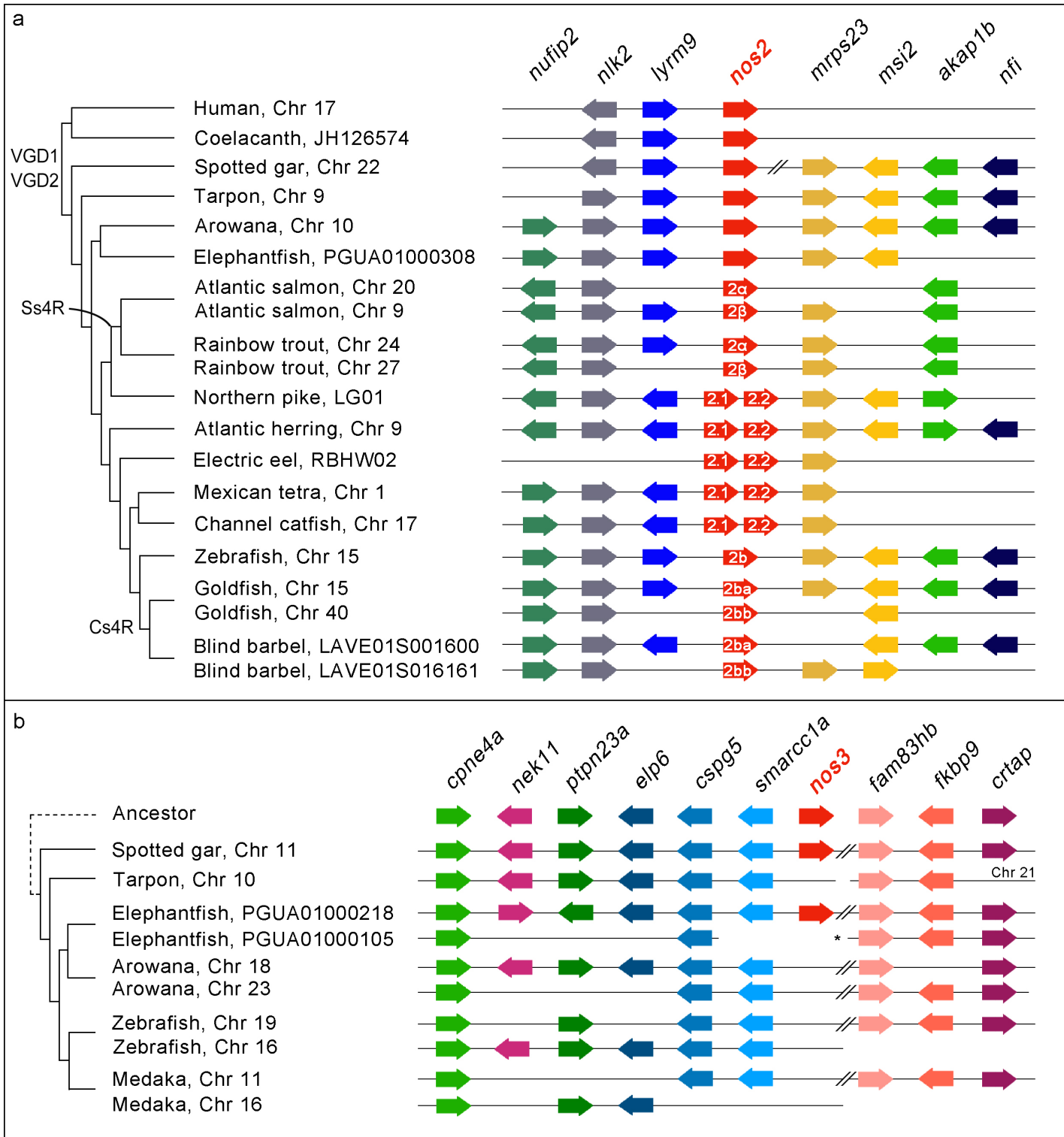

237 Figure 2. Conserved microsynteny of nos 2 and nos3. a, The nos2 paralogs derived 238 from different duplication modalities: carp-specific genome duplication (Cs4R) (nos2ba and $239 n o s 2 b b$ in the goldfish and blind golden-line barbel); salmonid-specific genome duplication 240 (Ss4R) (nos2 $\alpha$ and nos $2 \beta$ in the Atlantic salmon and rainbow trout); tandem gene 241 duplication occurred independently in five lineages (nos2.1 and nos2.2 in the northern 242 pike, Atlantic herring, electric eel, Mexican tetra and channel catfish). An additional nos2 243 duplicate (nos2a) is present in cyprinids (zebrafish, goldfish, and blind barbel). b, A 244 conserved synteny map of genomic regions around the nos 3 gene locus highlights the loss in Clupeocephala (including zebrafish and medaka), and in Osteoglossomorpha 
246 (arowana). Genes are represented as arrows and are coulor coded according to their 247 orthology and ohnology. The direction of arrows indicates transcription orientation. The 248 symbol // indicates a long-distance on a chromosome. The asterisk indicates scaffold 72 of 249 the freshwater elephantfish genome [24].

\section{Expression of nos in vertebrate developing gills}

253 Spotted gar is an important emerging model organism because it represents an 254 evolutionary bridge between teleosts and tetrapods that facilitates cross-species 255 comparisons. The gar genome is slowly evolving compared to that of teleosts and has 256 preserved a more ancient structural organization [29]. Therefore, we examined the 257 expression patterns of nos genes during gar development. As expected, based from the 258 literature, nos1 was expressed in several regions of the developing nervous system 259 (Supplementary Fig. 2). In contrast, nos2 expression was not detected during the 260 developmental stages covered in the present study, i.e., from 4 to 14 days post fertilization 261 (dpf). Unexpectedly, the expression of nos3 was first detected in gar embryos in the 262 pharyngeal area at $4 \mathrm{dpf}$ (Fig. 3a-b) and increased at $6 \mathrm{dpf}$ (Fig. 3c-d). At $7 \mathrm{dpf}$, embryos 263 showed clear nos3 expression in developing arches III, IV, and V (Fig. 3e-g). Later, at 11 $264 \mathrm{dpf}$, the positive signal is localized in gill filaments (Fig. 3i-k). Histological sections 265 highlighted the presence of nos3 in the epithelium of branchial lamellae (Fig. 3I), also 266 confirmed by the signal in gill structures in an advanced stage of maturation in $14 \mathrm{dpf}$ 267 juveniles (Fig. 3m-p). 


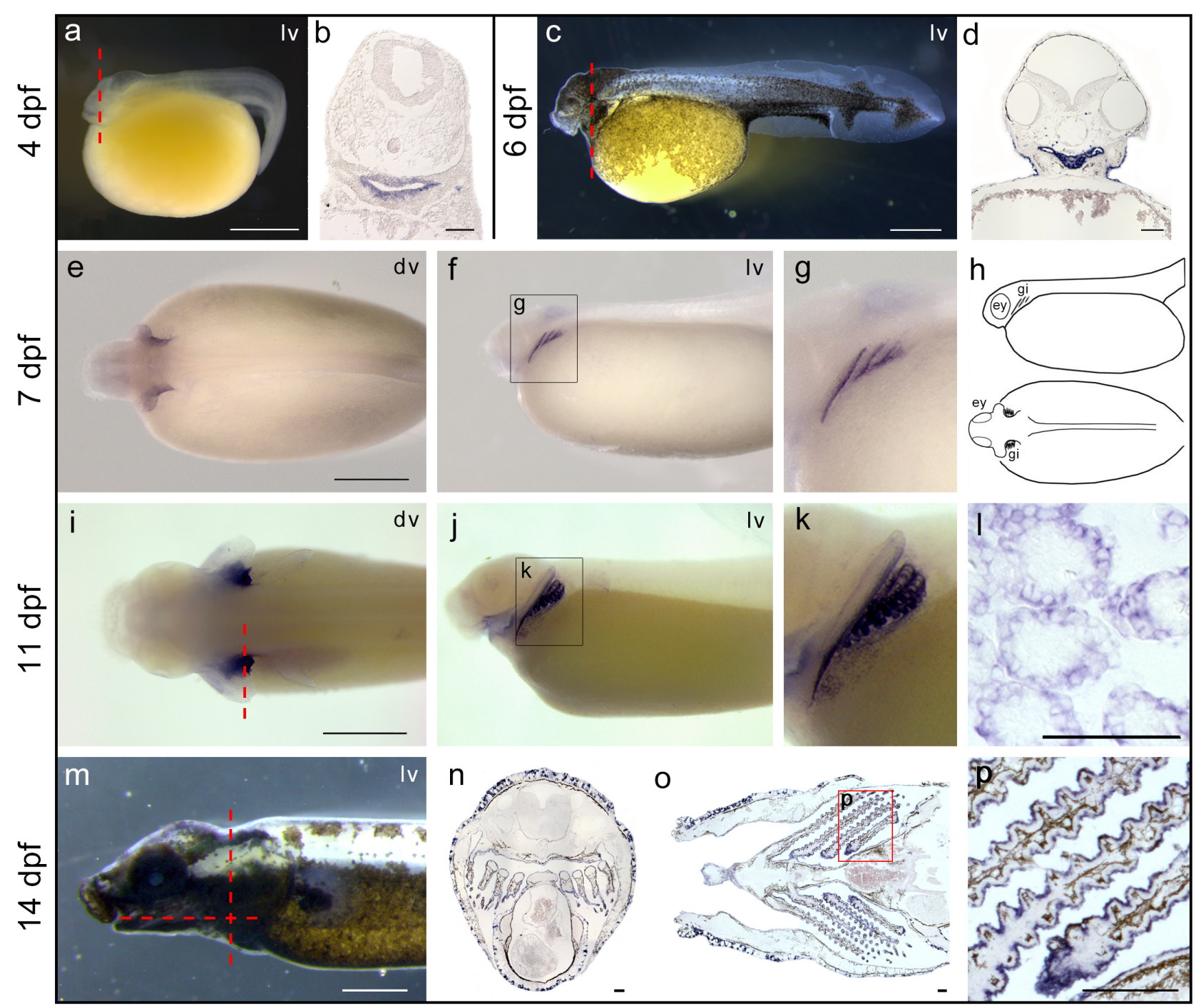

Figure 3. Spotted gar nos3 localization during development. Expression of nos 3 is

271 localized in the pharyngeal area in $4 \mathrm{dpf}$ (a-b) and $6 \mathrm{dpf}$ (c-d) embryos, in pharyngeal

272 arches in $7 \mathrm{dpf}$ larvae (e-g) schematized in (h), in developing gills in $11 \mathrm{dpf}$ late larvae (i-I),

273 and in gill lamellae in $14 \mathrm{dpf}$ juveniles (m-p). Coronal (n) and transversal section (o)

274 planes are indicated with a red dashed line in $(\mathbf{m})$. Abbreviations: ey, eye; gi, gill; dv,

275 dorsal view; Iv, lateral view. Scale bar is $1 \mathrm{~mm}$ in a, c, e, i, m; $100 \mu \mathrm{m}$ in b, d, I, n, o, p.

278 The detection of nos3 transcripts in gills of spotted gar and the established involvement of

279 NO gas in osmoregulatory control and vascular motility in gills of numerous teleosts [30-

28035 ] prompted us to investigate whether a similar nos expression patterns occurred in 281 developing gills of other fish species. We investigated nos expression in the sterlet 
sturgeon $A$. ruthenus and the bichir $P$. senegalus, members of early-branching groups of ray-finned fishes [21]. Moreover, we similarly investigated nos expression in the chondrichthyan cloudy catshark S. torazame to infer the ancestral expression condition among gnathostomes. Unlike gar, we discovered that nos3 was not expressed in gills of other species analysed in this work (Supplementary Fig. 2), thus raising questions about whether nos 3 expression in gills represents an oddity of holosteans or gars. Surprisingly, nos 1 and nos 2 were expressed in gills of sturgeon, bichir, and shark. In particular, nos2 was expressed in the branchial area of the sterlet sturgeon (Fig. 4a-c) and bichir embryos (Fig. 4d-f), while nos1 is expressed in gills of catshark embryos (Fig. 4g-i).

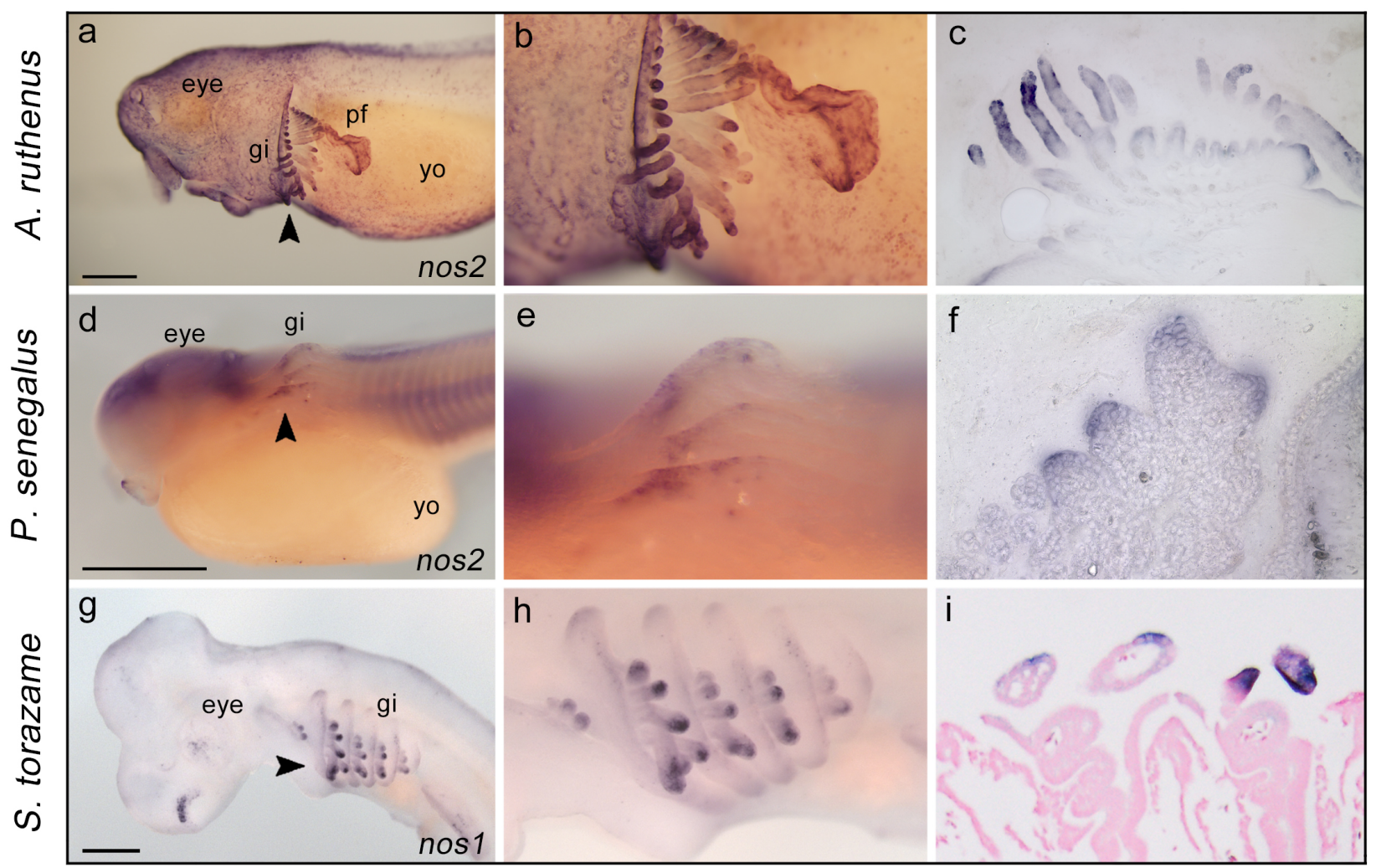

Figure 4. Expression of nos genes in developing gills of sterlet sturgeon, bichir, and shark embryos. The expression of nos 2 in the gills of sterlet sturgeon Acipenser ruthenus

295 (14 mm stage, a-c) and bichir Polypterus senegalus (stage 31, d-e); nos1 in the shark 296 Scyliorhinus torazame (stage 27, g-i). Higher magnification views of the gill structure of a, $\mathbf{d}, \mathbf{g}$ are shown in $\mathbf{b}, \mathbf{e}, \mathbf{h}$, respectively. The arrowheads indicate sectioning plane $(\mathbf{a}, \mathbf{d}, \mathbf{g})$ : 
transversal sections (c, f, $50 \mu \mathrm{m})$ and frontal section (I, $10 \mu \mathrm{m})$. Abbreviations: gi, gill; yo, yolk; pf, pectoral fin. Scale bar in a, d, $g$ is $0.5 \mathrm{~mm}$.

Our results show that nos paralogs are expressed in pharyngeal arches and gills in both actinopterygians and chondrichthyans. These findings lead us to question whether nos expression in gills could be a conserved feature also in sarcopterygians, and in particular

in amphibians that use gills for gas exchange. Therefore, to investigate the presence of nos transcripts in amphibia, we chose the neotenic axolotl Ambystoma mexicanum because it retains functional external gills throughout life. Gene expression analysis by qPCR revealed that nos 1 and nos 2 are almost not detectable in adult axolotl gills, while nos3 turned out to be highly expressed in gill structures (Supplementary Fig. 3). Therefore, we conclude that nos expression in gills is a conserved feature in neotenic amphibian assayed, previously observed exclusively in fishes.

\section{Expression of nos genes in the lamprey}

314 In cyclostomes (jawless vertebrates, including lampreys and hagfish), cartilaginous and 315 bony gnathostomes (jawed vertebrates), gills are endoderm-derived structures, pointing to 316 a single origin of pharyngeal gills before the divergence of these vertebrate lineages $317[36,37]$. The two lamprey nos paralogs, nos $A$, and nos $B$, display an unresolved orthology 318 relationship with their gnathostomes nos1, nos2, and nos3 (Fig. 1). To assess whether nos $A$ and nos $B$ are expressed in gills during embryogenesis, we performed whole-mount in situ hybridization experiments at different embryonic stages. We found that lamprey nosA was expressed in several tissues, including the brain, dorsal midline epidermis,

322 tailbud, mouth, and cloaca, but not in gills (Fig. 5a-b). Conversely, the lamprey nosB 323 paralog showed restricted expression in the developing mouth, specifically in the cheek 
324 process, including upper and lower lip regions (Fig. 5c-d). These results show that in the arctic lamprey, neither of the two nos paralogs is expressed in immature or mature gills, suggesting a fundamental difference in the role of nos genes in jawless and jawed vertebrates.

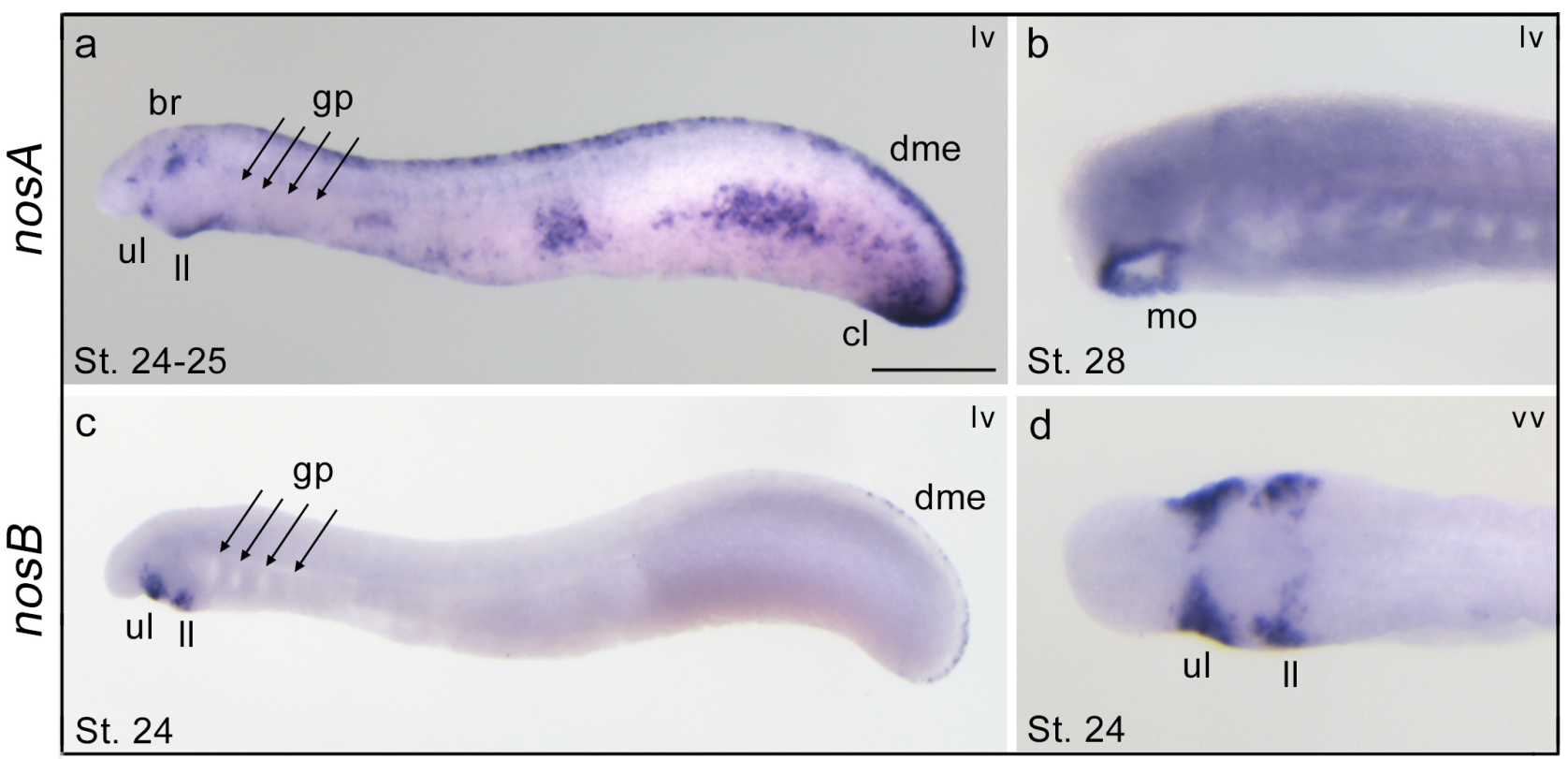

Figure 5. Expression patterns of nos $A$ and nos $B$ in larvae of the arctic lamprey. At stage 24-25 the nosA is expressed in the brain, mouth, upper and lower lip, dorsal midline epidermis, and cloaca (a). At stage 28, nosA expression is restricted to the mouth (b). The $n o s B$ is exclusively expressed in the cheek process, consisting of upper and lower lips (cd), and faint expression in the dorsal midline epidermis (c). Abbreviations: br, brain; cl, cloaca; dme, dorsal midline epidermis; gp, gill pouches; mo, mouth; II, lower lip; ul, upper lip; Iv, lateral view; vv, ventral view. Scale bar in a is $0.5 \mathrm{~mm}$.

\section{Discussion}

340 Actinopterygian fishes experienced one of the largest radiations in the animal kingdom and

341 their history represents a valuable resource for the formulation of hypotheses regarding 342 the evolution of vertebrate gene families. In this work, we employed data from recent 
343 genome projects to clarify and update the evolution of Nos family across vertebrates. We

344 performed a phylogenetic reconstruction using Nos protein sequences from key vertebrate 345 groups, including cyclostomes for which little information has previously been available. 346 Our phylogenetic analysis confirmed that Nos1 is ubiquitously present as single copy gene 347 across the gnathostome lineage, at least in the covered osteichthyan and chondrichthyan 348 species. Branch lengths of the Nos1 clade suggest a slow evolutionary rate throughout 349 vertebrate evolution in respect to the other two nos genes. Furthermore, our phylogenetic 350 data, complemented with syntenic analyses, highlighted for the first time a highly complex 351 scenario of Nos2 evolution, for which we suggest a nomenclature that attempts to 352 incorporate evolutionary origins into gene names. Previous analyses showed the presence 353 of two nos2 genes (nos2a and nos $2 b$ ) in zebrafish and goldfish $[38,39]$. Here we show the 354 presence of a nos2a paralog also in other two cyprinids, C. carpio and S. anshuiensis (Fig. 355 1a and Fig. 2a). nos2a and nos2b likely derive from an event of gene duplication that 356 occurred specifically at the stem of the group, and not related to the classic TGD. This 357 result is supported by synteny analysis since the chromosomal position of nos2a and 358 nos $2 b$ genes is not conserved (Fig. 2a and Supplementary Fig. 1), as it would be expected 359 if they were retained after a whole-genome duplication. On the other hand, the cyprinid 360 nos $2 b$ paralog independently duplicated in carps after the Cs4R [26], as the conserved 361 synteny suggests (Fig. 2a). In salmonids, synteny analysis also implies that the two Nos2 362 paralogs originated secondarily after the Ss4R (Fig. 2a) $[27,28]$. Here, we call these genes 363 nos2ba and nos $2 b b$ in carps to emphasize and clarify their relationships to zebrafish 364 genes, and nos $2 \alpha$ and nos $2 \beta$ in salmonids to indicate their distinct evolutionary origin. 365 Additionally, the present work shows that nos2 has undergone several independent 366 lineage-specific tandem gene duplication events (nos2.1 and nos2.2) (Fig. 2a). The search 367 of nos2 in available fish genomes, covering all main groups, failed to find it in any 
368 Neoteleostei, and for this reason, we hypothesized a nos2 gene loss event occurred in stem Neoteleostei (Fig. 1 and Fig. 6). It is worth mentioning that NO produced upon

370 stimulation of the inducible nos (nos2) is considered one of the most versatile players of 371 the immune system against infectious diseases, autoimmune processes and chronic 372 degenerative diseases [4,40]. For this reason, it would be important in the future to 373 investigate the impact of Nos2 loss on the immune response in Neoteleostei and if any 374 compensatory mechanisms occurred through the activation of other nos paralogs. In 375 addition, Nos2 is the only nos gene with retained duplicates in vertebrates, therefore, it 376 would also be important to understand if nos2 duplicates underwent neofunctionalization 377 or subfunctionalization, thus providing new functional features to the organism.

378 Concerning nos3, our understanding of its evolutionary history had a twist with the finding 379 of a nos3 ortholog in the spotted gar genome [20], proving that the previously postulated actinopterygian-specific loss of nos3 was an incorrect inference. Fostered by this discovery, we specifically searched for the presence of nos3 orthologs in a wide range of 382 fish species to infer the ancestral condition. We identified a nos 3 gene in bowfin, thus 383 confirming the presence of nos3 in the other reference genus of the holostean clade, in 384 addition to gar (Fig. 1a and Fig. 6). Furthermore, the presence of nos3 in genomes of bichir and sterlet sturgeon, which diverged prior to the teleostean and holostean split, confirmed the hypothesis that nos3 was already present in the common ancestor of extant 387 osteichthyans, rather than an innovation of tetrapods [8] or neopterygians (holosteans plus 388 teleosts) [20] (Fig. 6). We did not find nos3 gene in the tarpon M. cyprinoides genome (Fig. 389 2b), and to date, the limited genomic and transcriptomic data of eels, congers, and morays 390 cannot endorse the presence of a nos3 in Elopomorpha. Therefore, more genome 391 sequences are necessary to confirm its absence in this key group. We also did not find 392 nos3 in any fish from Clupeocepahala (non-elopomorph and non-osteoglossomorph 
393 teleosts; Fig. 1a and Fig. 2b) suggesting that a loss event took place in the common 394 ancestor of clupeocephalans (Fig. 1c and Fig. 2b). Notably, we found a nos3 gene in the 395 osteoglossomorph elephantfish P. kingsleyae (Fig. 1a and Fig. 2b), and it allowed us to 396 confirm that the loss of nos3 did not occur in the last common teleost ancestor, as 397 previously thought [20]. These findings suggest instead the following evolutionary scenario 398 for the nos3 gene: first, since we only find a maximum of one nos3 gene in those cases where it is present, we assume that one of the two TGD ohnologs was immediately lost after the TGD, and the other one was retained. This nos3 gene was then lost in the 401 ancestors of elopomorphs -although further research is needed to confirm this- and 402 clupeocephalans independently in separate events (Fig. 6).

403 The discovery of nos3 in sharks (S. torazame in this study) suggests that the origin of nos3 404 predates the divergence of gnathostomes and that three distinct nos paralogs were 405 already present in the last common ancestor of gnathostomes (Fig. 6), likely originating 406 after the two rounds of whole-genome duplication that took place during early vertebrate 407 evolution (VGD1 and VGD2, 2R hypothesis) [41]. The origin of nos genes is, in fact, 408 supported by the linkage to the evolutionarily conserved Hox gene clusters and several 409 other syntenic genes (Fig. 6b and Supplementary Fig. 4). Under this scenario, then a 410 fourth nos gene (putative nos4) should have existed but was apparently lost early in the 411 gnathostome evolution (Fig. 6a).

412 The apparent lack of nos genes in some vertebrate lineages remains to be clarified, such 413 as the absence of nos3 in coelacanth L. chalumnae (an extant basally diverging 414 sarcopterygian), in arowana S. formosus (an osteoglossomorph), and in elopomorph 415 fishes. In the future, further genomic projects will surely fill these gaps in our 416 understanding of this fascinating gene family. 
a

Ancestral gnathostome (VGD1 \& VGD2)

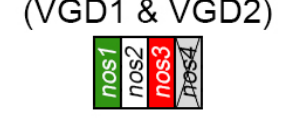

b

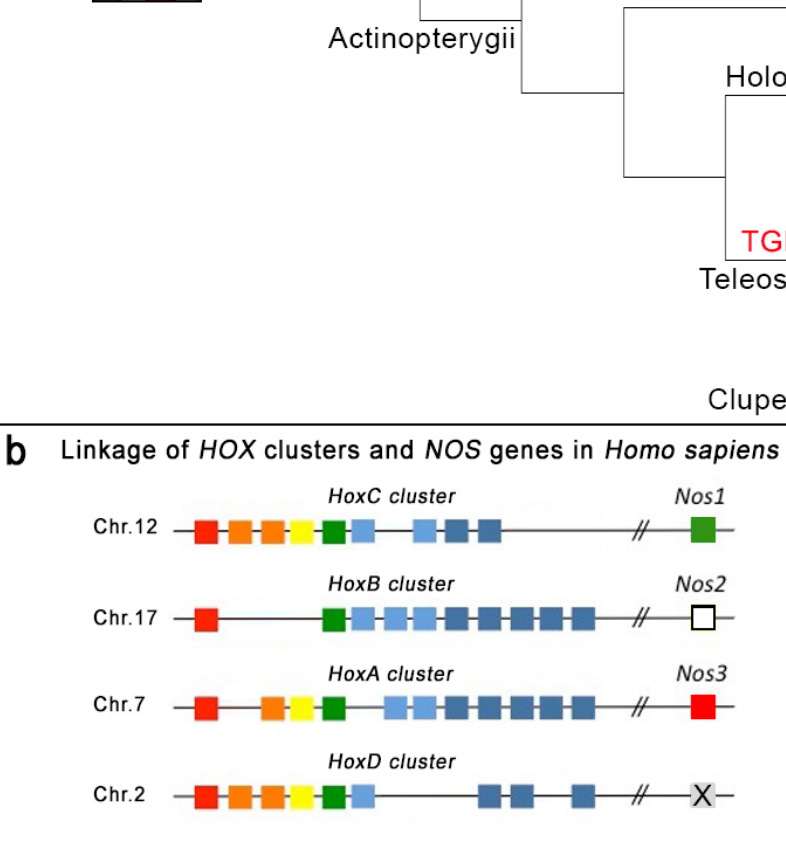

ШD Chondrichthyes

$\square$ Tetrapods

]? Coelacanthiformes

$\square$ Polypteriformes

प1 Acipenseriformes

\section{$\square$ Amiiformes}

D Lepisosteiformes

??? Elopomorphs

Ш? Osteoglossidae

-11 Mormyridae

DI Otomorpha

Protacanthopterygii

Neoteleosts

Figure 6. Nos evolution in light of recent gene findings in vertebrates. The proposed evolution of nos genes in gnathostomes (a) supposes an ancestral loss of a predicted 421 fourth nos gene (grey box), based on the linkage of human nos and Hox clusters (b). Loss 422 of nos3 occurred in stem Clupeocephala and loss of nos2 in stem Neoteleostei (a). 423 Species-specific nos2 duplications occurred in some Otomorpha, including Cyprinidae and 424 Characidae families.

The importance of NO in the ontogeny and function of vertebrate gills has already been documented in the context of physio-pharmacological studies, primarily using inhibitors of Nos activity. In gills, NO acts as a paracrine and endocrine vasoactive modulator and, 430 therefore, plays a crucial role in the distribution of oxygenated blood [42]. Moreover, NO 431 has an osmoregulatory function controlling the movement of ions across the gill epithelium 432 [33,43-45], and represents an important molecular component of the immune system 433 employed by macrophages to attack and destroy pathogens [46]. Nevertheless, 
434 documentation of Nos enzymatic activity in fish gills has relied exclusively upon techniques 435 unable to discriminate among individual Nos proteins, such as NADPH-diaphorase activity 436 and immunolocalization with heterologous mammalian antibodies [42,44,45,47]. Therefore, 437 the detected enzymatic activity has for a long time been indicated generically as 'Nos-like'. 438 Here we used a different approach based on mRNA transcript detection methodology, 439 which unequivocally distinguishes different genes, and showed, for the first time, that 440 indeed nos genes are expressed in gills during development in various vertebrates. 441 However, surprisingly different Nos paralogs are expressed in gills in different animals 442 tested: nos1 in shark, nos2 in bichir and sterlet sturgeon, and nos3 in spotted gar. The 443 most parsimonious hypothesis to explain this result is that the ancestral nos gene had a 444 number of roles in gills, immune system, brain, and other organs that was controlled by 445 separate regulatory elements and, due to subfunctionalization after the vertebrate $2 \mathrm{R}$ 446 (according to the Duplication-Degeneration-Complementation (DDC) model) [48], these 447 physiological roles partitioned to different nos ohnologs as lineages diverged and 448 reciprocal loss of the gill expression function occurred in a lineage-specific way. Further 449 support to this hypothesis comes from the identification of nos1-positive cells in gill of 450 zebrafish at $5 \mathrm{dpf}$, in addition to brain, eye, periderm and NaK ionocytes, according to the 451 recently released developmental single-cell transcriptome atlas [49] (Supplementary Fig. $4525)$.

453 Additionally, to corroborate the involvement of NO in normal gill physiology, we searched 454 for nos expression in gills of a paedomorphic amphibian, the Mexican axolotl, which 455 maintains gill structures in adulthood. Taking into account the different evolutionary and 456 developmental origin of internal and external gills [50], the conservation of nos3 457 expression in gills indicated that the NO signaling system could be indeed fundamental for 458 the physiology and development of this structure in the axolotl, and perhaps generally in 
459 pre-metamorphic amphibians. Therefore, our data obtained from established and 460 emerging model species highlighted that the expression of at least one nos gene has a 461 functional role in gnathostome gills.

462 Recently, a single origin of pharyngeal gills predating the divergence of cyclostomes and 463 gnathostomes was suggested [36]. Therefore, we investigated whether either of the two 464 arctic lamprey nos paralogs is expressed in developing gills, but found them expressed 465 mainly in the nervous system, mouth and pharynx, similarly to the expression pattern 466 previously reported in the cephalochordate amphioxus [51,52]. Nevertheless, we found 467 neither of the two genes to be expressed in gills during lamprey development, leading us 468 to speculate that either the expression of nos genes in gills was acquired in gnathostomes 469 after the divergence from cyclostomes, or alternatively, gill expression was a feature of 470 their last common ancestor but lost in the lamprey lineage. Future work on hagfish 471 embryology would be necessary to help solve this issue.

472 In conclusion, our findings pave the way for future studies that aim to investigate the 473 ontogenetic role of nitric oxide in gill development of aquatic vertebrates, possibly by loss474 of-function approaches using either Nos protein-specific chemical inhibitors or CRISPR475 Cas9 gene editing. From the perspective of the evolution of developmental mechanisms, it 476 would be interesting to understand more about the species-specific regulatory 477 mechanisms that drive different nos genes expression patterns in gills in different species

\section{Materials and Methods}

481 Sequence mining and phylogenetic analysis

482 Nos sequences used for evolutionary analyses were retrieved from NCBI 483 (https://www.ncbi.nlm.nih.gov/), Ensembl (www.ensembl.org/index.html), Skatebase 
484 (http://skatebase.org/) and DDBJ (https://www.ddbj.nig.ac.jp/index-e.html) databases, 485 using direct browser webpages or by downloading fully assembled genomes and 486 transcriptomes (see Supplementary Table 1 for accession numbers). The quality of protein 487 sequences was checked and, where needed, manually curated excluding from the dataset 488 partial or low blast score sequences. We used proteins from Homo sapiens, Anolis carolinensis and Xenopus tropicalis as internal references, and two non-vertebrate chordates as outgroups: the cephalochordate Branchiostoma lanceolatum NosA, NosB and NosC, and the tunicate Ciona robusta Nos.

492 Nos sequences from bowfin (A. calva) were obtained from a draft genome assembly [23]. 493 Lamprey nosA and nosB genes were obtained by TBLASTN v2.2.31+ searches [53] from 494 the v1.0 draft genome of arctic lamprey L. camtschaticum [25] and the germ line draft 495 genome of sea lamprey P. marinus [54]. Initial predictions were extended, corrected, and confirmed by RACE PCRs in the case of $L$. camtschaticum. Both $P$. marinus nosA and nosB were manually curated using Wise2 [55]. The single nos gene sequence from the 498 inshore hagfish E. burgeri was obtained from a de novo transcriptome assembly [56]. 499 Sequences of nos1, nos2, and nos3 genes from the cloudy catshark S. torazame were 500 obtained from a de novo transcriptome assembly [56] employing TBLASTN v2.2.31+. A 501 partial nos1 sequence (g15096.t1) was found in the European eel $A$. anguilla 502 transcriptome database (EeelBase 2.0, http://compgen.bio.unipd.it/eeelbase/), but it was 503 deliberately excluded from the phylogenetic analyses because of alignment ambiguities, 504 probably due to gene assembly errors.

505 For phylogenetic analysis, Nos amino acid sequences were aligned using the MUSCLE 506 algorithm [57] as implemented in MEGAX (version 10.2.4) [58], with default parameters, 507 run in a MacOS 11.2.1 operating system and saved in FASTA format. The alignment was 508 trimmed by trimAl v1.2rev59 [59], using the '-automated1' parameter. The trimmed 
509 alignment was then formatted into a nexus file using readAl (bundled with the trimAl 510 package) (supplementary file S1). A Bayesian inference tree was constructed using 511 MrBayes v3.2.6 [60], under the assumption of an $L G+\mid+G$ evolutionary model. Two 512 independent MrBayes runs of 2,000,000 generations were performed, with four chains 513 each and a temperature parameter value of 0.05 . The tree was considered to have 514 reached convergence when the standard deviation stabilized under a value of $<0.01$. A 515 burn-in of $25 \%$ of the trees was performed to generate the consensus tree $(1,500,000$ 516 post-burnt-in trees).

\section{Synteny}

519 Conserved synteny analyses were manually performed on fish chromosomes or scaffolds. 520 With the aim of finding synteny blocks flanking the nos 2 and nos 3 orthologs, we employed 521 the Synteny Database (http://syntenydb.uoregon.edu/synteny_db/) [61,62]. Additional 522 information was retrieved in NCBI (https://www.ncbi.nlm.nih.gov/), Ensemble v.102 (http://www.ensembl.org/index.html) and Genomicus v100.01 (https://www.genomicus.biologie.ens.fr/genomicus-100.01/cgi-bin/search.pl) [61].

\section{Collection of embryos and tissues}

527 Spotted gar L. oculatus adult specimens were collected from the Atchafalaya River basin, 528 Louisiana (USA) and cultured in a $2 \mathrm{~m}$ diameter tank containing artificial spawning 529 substrate. Spawning was induced by injection of Ovaprim@ $(0.5 \mathrm{ml} / \mathrm{kg})$ and embryos were 530 raised in fish water (salinity $1 \mathrm{ppt}$ ) at $24^{\circ} \mathrm{C}$ in a $14 / 10 \mathrm{~h}$ light/dark cycle [63]. The

531 developmental staging was determined following hours or days post fertilization in addition 532 to morphological criteria [64]. 
533 Embryos of $L$. camtschaticum were obtained by artificial fertilization, cultured at a 534 temperature ranging between 9 and $12^{\circ} \mathrm{C}$, and staged as previously described $[65,66]$. 535 Embryos of S. torazame were obtained, cultured, and staged as previously described $536[67,68]$.

537 Bichir $P$. senegalus embryos were obtained from the breeding colony at the Department of 538 Zoology, Charles University, Prague (Czech Republic) by natural breeding. Embryos were 539 kept at $28^{\circ} \mathrm{C}$ and staged using Diedhiou and Bartsch (2009) guidelines [69]. Sterlet 540 sturgeon $A$. ruthenus embryos were obtained from the hatcheries of the Research Institute 541 of Fish Culture and Hydrobiology in Vodnany, University of South Bohemia (Czech 542 Republic). Embryos were raised in tanks containing E2 Pen/Strep zebrafish medium and 543 incubated at $17^{\circ} \mathrm{C}$ until the desired stages, according to Dettlaff and collaborators (1993) 544 [70]. Gill tissues from two adult axolotls (RRID:AGSC_110A) were collected under 545 benzocaine anesthesia (University of Kentucky, USA, IACUC protocol 2017-2580).

\section{Gene expression analysis by in situ hybridization}

548 For all species used in the present study, total RNA was isolated from a mix of embryo stages using the phenol-chloroform method with TRIzol (Thermo-Fisher Scientific). cDNA was synthesized from $1 \mu \mathrm{g}$ of total RNA using the SuperScript VILO cDNA Synthesis kit

551 (Thermo-Fisher Scientific). Primers for PCR amplification are listed in Supplementary 552 Table 2. Amplicons were cloned into the pGEM-T Easy Vector (Promega) and Sanger 553 sequenced. Antisense Digoxygenin-UTP riboprobes were synthesized using SP6 or T7 554 RNA polymerases and the DIG RNA Labeling kit (Roche).

555 Whole-mount in situ hybridization experiments were performed following protocols 556 previously described: spotted gar [71], bichir and sturgeon [72], lamprey [65], and shark 557 [73], with slight modifications. For spotted gar embryos at $7 \mathrm{dpf}$ (Long \& Ballard stage 24) 
558 and $11 \mathrm{dpf}$ (Long \& Ballard stage 28$)$, longer proteinase $\mathrm{K}(10 \mu \mathrm{g} / \mathrm{mL})$ digestion times were 559 performed, respectively 25 and 35 minutes at $24^{\circ} \mathrm{C}$. Moreover, endogenous melanin 560 pigment was removed using bleaching solution $\left[\left(3 \%\right.\right.$ hydrogen peroxide $\left(\mathrm{H}_{2} \mathrm{O}_{2}\right)$ and $1 \%$ 561 potassium hydroxide $(\mathrm{KOH})$ in distillate water $\left.\left(\mathrm{ddH}_{2} \mathrm{O}\right)\right]$ for a few minutes. For 14 dpf gar 562 embryos (Long \& Ballard stage 31), we performed in situ hybridizations on cryosections, 563 as previously described [74], including modifications reported in [75].

564 Transversal vibratome sections of bichir and sturgeon embryos (thickness $50 \mu \mathrm{m}$ ) were 565 made on whole-mount hybridized embryos upon embedding in 566 gelatin/albumin/glutaraldehyde [50]. Shark embryos were embedded in paraffin after 567 whole-mount in situ hybridization assays, and frontal sections (10 $\mu \mathrm{m})$ were obtained with 568 a microtome.

569 Whole-mount and sectioned preparations mounted on slides were imaged on Axio Imager 570 Z2 with Apotome 2 (Carl Zeiss), equipped with Axiocam 503 coulor digital camera and 571 Axio Vision software for analysis. Whole-mount bichir and sturgeon embryos were 572 photographed as Z-stacks using a motorized dissection microscope (Olympus SZX12) and 573 deep-focus images were generated by merging Z-stacks in QuickPhoto Micro.

\section{Real-time PCR}

576 Expression levels of nos genes in axolotl $A$. mexicanum gills were analysed by qPCR 577 using specific primers reported in Supplementary Table 2. The atpf51 gene was used as a 578 reference. RNA was isolated by performing a chloroform extraction and isopropanol precipitation. RNA was quantified using a Nanodrop and $1 \mu \mathrm{g}$ of total RNA was used to 580 generate cDNA using an Invitrogen Super-Script IV cDNA synthesis kit with oligo-dT 581 tailing. RT-qPCR was performed in triplicate with SYBER Master Mix on a LightCycler 96 
582 (Bio-Rad), using a 2-step amputation protocol $\left(95^{\circ} \mathrm{C}\right.$ for $10 \mathrm{sec}, 60^{\circ} \mathrm{C}$ for $\left.30 \mathrm{sec}\right)$ and 40 cycles. Data were analysed using the $\Delta \Delta \mathrm{CT}$ method.

\section{Data availability}

586 Accession numbers of protein sequences used in the phylogenetic analysis are available 587 in Supplementary Table 1. Primer sequences used for the synthesis of in situ hybridization 588 riboprobes and in quantitative real-time PCR experiments are given in Supplementary 589 Table 2.

\section{Acknowledgments}

592 The authors thank Allyse Ferrara and Quenton Fontenot, Louisiana State University 593 (USA), for their help in the generation of spotted gar embryos. The authors thank Fumiaki 594 Sugahara for his help in the interpretation of results in the arctic lamprey, Anna Pospisilova 595 for technical assistance with bichir and sturgeon in situ hybridizations, and Martin 596 Psenicka, Roman Franek, Michaela Fucikova, Marek Rodina, David Gela, and Martin 597 Kahanec for sterlet sturgeon spawns. A special thanks to Robert Cerny for the 598 establishment of the African bichirs colony at the Charles University in Prague.

599 Giovanni Annona was supported by the Research grant POR Campania FSE 2014/2020 600 (IT) and by the EMBO Short Term Fellowship (\# 6936) to visit the Postlethwait laboratory 601 in Oregon (USA) and for the field trip in Louisiana (USA). Jan Stundl is supported by the 602 European Union's Horizon 2020 research and innovation program under the Marie 603 Skłodowska-Curie grant agreement No. 897949. Vladimir Soukup is supported by the 604 Charles University Research Centre program No. 204069 and grant SVV260571/2020. 605 Randal Voss and the Ambystoma Genetic Stock Center are supported by the National 606 Institutes of Health, USA (P40OD019794). John H. Postlethwait is supported by the R01 
607 OD011116 grant from the US National Institutes of Health. Salvatore D'Aniello is 608 supported by the NOEVO grant from the Stazione Zoologica Anton Dohrn Napoli.

\section{Competing interests}

611 The authors declare no competing interests.

\section{References}

614 1. Koshland D. The molecule of the year. Science (80). 1992;258: 1861-1861.

615 2. Strijdom H, Chamane N, Lochner A. Nitric oxide in the cardiovascular system: a simple molecule with complex actions. Cardiovasc J Afr. 2009;20: 303-10.

3. Esplugues J V. NO as a signalling molecule in the nervous system. Br J Pharmacol. 2002;135: 1079-1095.

4. Bogdan C. Nitric oxide and the immune response. Nat Immunol. 2001;2: 907-916.

5. Knott AB, Bossy-Wetzel E. Nitric Oxide in Health and Disease of the Nervous System. Antioxid Redox Signal. 2009;11: 541-553.

6. Kamm A, Przychodzen P, Kuban-Jankowska A, Jacewicz D, Dabrowska AM, Oxide. 2019;93: 102-114.

7. Santolini J. What does Idquo NO-Synthase rdquo stand for ? Front Biosci. 2019;24:

8. Andreakis N, D'Aniello S, Albalat R, Patti FP, Garcia-Fernandez J, Procaccini G, et 2011;28: 163-179.

9. Berg DA, Belnoue L, Song H, Simon A. Neurotransmitter-mediated control of neurogenesis in the adult vertebrate brain. Development. 2013;140: 2548-2561. 
632 10. Steinert JR, Chernova T, Forsythe ID. Nitric Oxide Signaling in Brain Function, Dysfunction, and Dementia. Neurosci. 2010;16: 435-452.

11. Moncada S, Higgs EA. The discovery of nitric oxide and its role in vascular biology. Br J Pharmacol. 2006;147: S193-S201.

12. Namba T, Koike H, Murakami K, Aoki M, Makino H, Hashiya N, et al. Angiogenesis Induced by Endothelial Nitric Oxide Synthase Gene Through Vascular Endothelial Growth Factor Expression in a Rat Hindlimb Ischemia Model. Circulation. 2003;108: $2250-2257$.

13. Förstermann U, Sessa WC. Nitric oxide synthases: regulation and function. Eur Heart J. 2012;33: 829-837.

14. Postlethwait J, Amores A, Force A, Yan YL. The zebrafish genome. Methods Cell Biol. 1998;60: 149-163.

15. Amores A. Zebrafish hox Clusters and Vertebrate Genome Evolution. Science (80- ). 1998;282: 1711-1714.

16. Taylor JS, Van de Peer Y, Braasch I, Meyer A. Comparative genomics provides evidence for an ancient genome duplication event in fish. Schilling T, Wilson S,

17. Jaillon O, Aury J-M, Brunet F, Petit J-L, Stange-Thomann N, Mauceli E, et al. vertebrate proto-karyotype. Nature. 2004;431: 946-957.

18. Tota B, Amelio D, Pellegrino D, Ip YK, Cerra MC. NO modulation of myocardial

19. Agnisola C, Pellegrino D. Role of nitric oxide in vascular regulation in fish. Advances in Experimental Biology. 2007. pp. 293-310. 
657 20. Donald JA, Forgan LG, Cameron MS. The evolution of nitric oxide signalling in vertebrate blood vessels. J Comp Physiol B. 2015;185: 153-171.

21. Hughes LC, Ortí G, Huang Y, Sun Y, Baldwin CC, Thompson AW, et al. Comprehensive phylogeny of ray-finned fishes (Actinopterygii) based on transcriptomic and genomic data. Proc Natl Acad Sci. 2018;115: 6249-6254.

22. Du K, Stöck M, Kneitz S, Klopp C, Woltering JM, Adolfi MC, et al. The sterlet sturgeon genome sequence and the mechanisms of segmental rediploidization. Nat Ecol Evol. 2020;4: 841-852.

23. Thompson A, Hawkins M, Parey E, Wcisel D, Ota T, Kawasaki K, et al. The genome

24. Gallant JR, Losilla M, Tomlinson C, Warren WC. The genome and adult somatic transcriptome of the mormyrid electric fish paramormyrops kingsleyae. Genome Biol Evol. 2017;9: 3525-3530.

25. Mehta TK, Ravi V, Yamasaki S, Lee AP, Lian MM, Tay B-H, et al. Evidence for at least six Hox clusters in the Japanese lamprey (Lethenteron japonicum). Proc Natl Acad Sci. 2013;110: 16044-16049.

27. Berthelot C, Brunet F, Chalopin D, Juanchich A, Bernard M, Noël B, et al. The rainbow trout genome provides novel insights into evolution after whole-genome duplication in vertebrates. Nat Commun. 2014;5: 3657.

26. Xu P, Xu J, Liu G, Chen L, Zhou Z, Peng W, et al. The allotetraploid origin and asymmetrical genome evolution of the common carp Cyprinus carpio. Nat Commun. 2019;10: 4625 .

28. Lien S, Koop BF, Sandve SR, Miller JR, Kent MP, Nome T, et al. The Atlantic salmon genome provides insights into rediploidization. Nature. 2016;533: 200-205. 
682 29. Braasch I, Gehrke AR, Smith JJ, Kawasaki K, Manousaki T, Pasquier J, et al. The spotted gar genome illuminates vertebrate evolution and facilitates human-teleost comparisons. Nat Genet. 2016;48: 427-437.

30. Gibbins IL, Olsson C, Holmgren S. Distribution of neurons reactive for NADPHdiaphorase in the branchial nerves of a teleost fish, Gadus morhua. Neurosci Lett. 1995;193: 113-116.

31. Mauceri A, Fasulo S, Ainis L, Licata A, Rita lauriano E, Martfnez A, et al. Neuronal

32. Fritsche R, Schwerte T, Pelster B. Nitric oxide and vascular reactivity in developing zebrafish, Danio rerio. Am J Physiol Integr Comp Physiol. 2000;279: R2200-R2207.

33. Evans DH. Cell signaling and ion transport across the fish gill epithelium. J Exp Zool. 2002;293: 336-347.

34. Haraldsen L, Söderström-Lauritzsen V, Nilsson GE. Oxytocin stimulates cerebral blood flow in rainbow trout (Oncorhynchus mykiss) through a nitric oxide dependent

35. Pellegrino D, Sprovieri E, Mazza R, Randall D., Tota B. Nitric oxide-cGMP-mediated vasoconstriction and effects of acetylcholine in the branchial circulation of the eel. Comp Biochem Physiol Part A Mol Integr Physiol. 2002;132: 447-457.

36. Gillis JA, Tidswell ORA. The Origin of Vertebrate Gills. Curr Biol. 2017;27: 729-732.

37. Warga RM, Nüsslein-Volhard C. Origin and development of the zebrafish endoderm. Development. 1999;126: 827-38. cavity. Dev Dyn. 2008;237: 1662-1667. 
39. Lepiller S, Franche N, Solary E, Chluba J, Laurens V. Comparative analysis of zebrafish nos2a and nos2b genes. Gene. 2009;445: 58-65.

40. Lind M, Hayes A, Caprnda M, Petrovic D, Rodrigo L, Kruzliak P, et al. Inducible nitric oxide synthase: Good or bad? Biomed Pharmacother. 2017;93: 370-375.

41. Dehal P, Boore JL. Two Rounds of Whole Genome Duplication in the Ancestral Vertebrate. Holland P, editor. PLoS Biol. 2005;3: e314.

42. Tota B, Amelio D, Cerra MC, Garofalo F. The morphological and functional significance of the NOS/NO system in the respiratory, osmoregulatory, and contractile organs of the African lungfish. Acta Histochem. 2018;120: 654-666.

43. Tipsmark $\mathrm{CK}$. Regulation of $\mathrm{Na}+/ \mathrm{K}+-\mathrm{ATP}$ ase activity by nitric oxide in the kidney and gill of the brown trout (Salmo trutta). J Exp Biol. 2003;206: 1503-1510.

44. Ebbesson LOE. Nitric oxide synthase in the gill of Atlantic salmon: colocalization with and inhibition of Na+,K+-ATPase. J Exp Biol. 2005;208: 1011-1017.

45. Hyndman KA, Choe KP, Havird JC, Rose RE, Piermarini PM, Evans DH. Neuronal nitric oxide synthase in the gill of the killifish, Fundulus heteroclitus. Comp Biochem Physiol Part B Biochem Mol Biol. 2006;144: 510-519.

46. Campos-Perez JJ, Ward M, Grabowski PS, Ellis AE, Secombes CJ. The gills are an important site of iNOS expression in rainbow trout Oncorhynchus mykiss after challenge with the Gram-positive pathogen Renibacterium salmoninarum. Immunology. 2000;99: 153-161.

47. Mistri A, Kumari U, Mittal S, Mittal AK. Immunohistochemical localization of nitric oxide synthase (NOS) isoforms in epidermis and gill epithelium of an angler catfish, Chaca chaca (Siluriformes, Chacidae). Tissue Cell. 2018;55: 25-30.

48. Force A, Lynch M, Pickett FB, Amores A, Yan YL, Postlethwait J. Preservation of duplicate genes by complementary, degenerative mutations. Genetics. 1999;151: 
$1531-45$.

49. Farnsworth DR, Saunders LM, Miller AC. A single-cell transcriptome atlas for zebrafish development. Dev Biol. 2020;459: 100-108.

50. Stundl J, Pospisilova A, Jandzik D, Fabian P, Dobiasova B, Minarik M, et al. Bichir external gills arise via heterochronic shift that accelerates hyoid arch development. Elife. 2019;8: e43531.

51. Annona G, Caccavale F, Pascual-Anaya J, Kuratani S, De Luca P, Palumbo A, et al. Nitric Oxide regulates mouth development in amphioxus. Sci Rep. 2017;7: 8432.

52. Caccavale F, Annona G, Subirana L, Escriva H, Bertrand S, D'Aniello S. Crosstalk between Nitric Oxide and Retinoic Acid pathways is essential for amphioxus pharynx development. bioRxiv. 2020.

53. Altschul S. Gapped BLAST and PSI-BLAST: a new generation of protein database search programs. Nucleic Acids Res. 1997;25: 3389-3402.

54. Smith JJ, Timoshevskaya N, Ye C, Holt C, Keinath MC, Parker HJ, et al. The sea lamprey germline genome provides insights into programmed genome rearrangement and vertebrate evolution. Nat Genet. 2018;50: 270-277.

55. Madeira F, Park YM, Lee J, Buso N, Gur T, Madhusoodanan N, et al. The EMBLEBI search and sequence analysis tools APIs in 2019. Nucleic Acids Res. 2019;47: W636-W641.

56. Pascual-Anaya J, Sato I, Sugahara F, Higuchi S, Paps J, Ren Y, et al. Hagfish and lamprey Hox genes reveal conservation of temporal colinearity in vertebrates. Nat Ecol Evol. 2018;2: 859-866.

57. Edgar RC. MUSCLE: multiple sequence alignment with high accuracy and high throughput. Nucleic Acids Res. 2004;32: 1792-1797.

58. Kumar S, Stecher G, Li M, Knyaz C, Tamura K. MEGA X: Molecular Evolutionary 
Genetics Analysis across Computing Platforms. Battistuzzi FU, editor. Mol Biol Evol. 2018;35: 1547-1549.

59. Capella-Gutierrez S, Silla-Martinez JM, Gabaldon T. trimAl: a tool for automated alignment trimming in large-scale phylogenetic analyses. Bioinformatics. 2009;25: 1972-1973.

60. Ronquist F, Teslenko M, van der Mark P, Ayres DL, Darling A, Höhna S, et al. MrBayes 3.2: Efficient Bayesian Phylogenetic Inference and Model Choice Across a

62. Catchen JM, Conery JS, Postlethwait JH. Automated identification of conserved synteny after whole-genome duplication. Genome Res. 2009;19: 1497-1505.

61. Nguyen NTT, Vincens P, Roest Crollius H, Louis A. Genomicus 2018: karyotype evolutionary trees and on-the-fly synteny computing. Nucleic Acids Res. 2018;46:

63. Braasch I, Guiguen Y, Loker R, Letaw JH, Ferrara A, Bobe J, et al. Connectivity of vertebrate genomes: Paired-related homeobox (Prrx) genes in spotted gar, basal teleosts, and tetrapods. Comparative Biochemistry and Physiology Part - C: Toxicology and Pharmacology. 2014. pp. 24-36.

64. Long WL, Ballard WW. Normal embryonic stages of the longnose gar, Lepisosteus osseus. BMC Dev Biol. 2001;1: 1-8.

65. Sugahara F, Murakami Y, Kuratani S. Gene Expression Analysis of Lamprey Embryos. In Situ Hybridization Methods. 2015. pp. 263-278.

66. Tahara Y. Normal stages of development in the lamprey, Lampetra reissneri (Dybowski). Zoolog Sci. 1988;5: p109-118.

67. Adachi N, Kuratani S. Development of head and trunk mesoderm in the dogfish, Scyliorhinus torazame : I. Embryology and morphology of the head cavities and 
related structures. Evol Dev. 2012;14: 234-256.

68. Ballard WW, Mellinger J, Lechenault $\mathrm{H}$. A series of normal stages for development of Scyliorhinus canicula, the lesser spotted dogfish (Chondrichthyes: Scyliorhinidae). J Exp Zool. 1993;267: 318-336.

69. Diedhiou S, Bartsch P. Staging of the Early Development of Polypterus (Cladistia: Actinopterygii). Development of Non-teleost Fishes. Science Publishers; 2009. pp. 104-169.

70. Dettlaff TA, Ginsburg AS, Schmalhausen OI. Sturgeon Fishes - Developmental Biology and Aquaculture. 1st ed. Berlin, Heidelberg: Springer Berlin Heidelberg; 1993.

71. Jowett T, Yan Y-L. Double fluorescent in situ hybridization to zebrafish embryos. Trends Genet. 1996;12: 387-389.

72. Minarik M, Stundl J, Fabian P, Jandzik D, Metscher BD, Psenicka M, et al. Pre-oral gut contributes to facial structures in non-teleost fishes. Nature. 2017;547: 209-212.

73. Adachi N, Takechi M, Hirai T, Kuratani S. Development of the head and trunk mesoderm in the dogfish, Scyliorhinus torazame : II. Comparison of gene expression between the head mesoderm and somites with reference to the origin of the vertebrate head. Evol Dev. 2012;14: 257-276.

74. Strähle U, Blader P, Adam J, Ingham PW. A simple and efficient procedure for nonisotopic in situ hybridization to sectioned material. Trends Genet. 1994;10: 75-76.

75. Jowett T, Ingham PW, Henrique D, Lettice L, Wilkinson D, Yan YL. An EMBO practical course. Univ Newcastle, Newcastle. 1995; 50. 